\title{
Classifying Finite Simple Groups and 2-Fusion Systems"
}

\section{by Michael Aschbacher ${ }^{\dagger}$}

\section{Introduction}

I hope to do a number of things in this expository article. First, I want to tell you a bit about the finite simple groups, and try to give you a vague idea of how they are classified. In a moment I'll begin that discussion with some motivation. Second, the proof of the classification is lengthy and complex, so it is important to try and simplify the existing proof. There are several ongoing efforts in that direction; I'm thinking in particular of the ambitious programs of Gorenstein, Lyons, and Solomon (cf. [GLS1]) and of Meierfrankenfeld, Stellmacher, and Stroth. I also have a program to simplify part of the proof of the classification. My approach involves carrying out most of the proof in the category of fusion systems, not in the category of groups. The latter part of the article discusses that program. In addition as fusion systems play such a large role in the program, before describing the program I'll first need to give a quick tutorial on fusion systems.

So we now have an idea of where we are headed: we begin with a discussion of why the finite simple groups and their classification are important. Then we move to a quick overview of the proof of the classification of the finite simple groups. This is followed by an introduction to the theory of fusion systems. Finally there is a discussion of a program that would, first, classify most simple 2-fusion systems of component type, and then, second, use the theorem on fusion systems to simplify that part of the classification of the finite simple groups dealing with groups of component type.

\footnotetext{
* This work was partially supported by DMS NSF-1265587 and DMS NSF-0969009.

† California Institute of Technology, Pasadena, Calif., U.S.A. E-mail: asch@its.caltech.edu
}

\section{Finite Simple Groups}

Recall a group $G$ is simple if 1 and $G$ are the only normal subgroups of $G$. Equivalently, $G$ and 1 are the only homomorphic images of $G$.

Consider subnormal series

$$
1=G_{0} \triangleleft G_{1} \triangleleft \cdots \triangleleft G_{n}=G
$$

for $G$ and the factors $\left(G_{i+1} / G_{i}: 0 \leq i<n\right)$ of the series. A subnormal subgroup of $G$ is a subgroup contained in some subnormal series. In a group with a chain condition on subnormal subgroups (like a finite group), there are maximal such series, and those are precisely the series in which all the factors are simple. These maximal series are called the composition series for $G$, and the family of factors of such a series is called the family of composition factors of $G$. By the JordanHolder Theorem the composition factors are independent of the composition series.

Thus the simple groups are the building blocks of finite group theory, analogous to the primes in arithmetic, in that each finite group is built from its composition factors. However unlike unique factorization in arithmetic, the composition factors of $G$ don't uniquely determine $G$; instead there is also:

The Extension Problem. Given groups $X$ and $Y$ determine the groups $G$ possessing a normal subgroup $H$ with $H \cong X$ and $G / H \cong Y$.

In the late nineteenth century, Holder proposed that one should adopt a two-step program in studying finite groups:

1. Classify the finite simple groups.

2. Solve the Extension Problem.

It developed that it is possible to classify the simple groups, although just barely. But the Extension 
Problem seems to be just too complex, except in special cases; put another way, attempting to classify all finite groups is not the right problem. Instead it turns out that, for some reason, it seems to be possible to reduce many (most?) problems in finite group theory to some corresponding problem on some class of minimal groups, which are usually close to simple. Then, given the classification together with enough information about the simple groups on our list, it is often possible to solve the original problem. In other words we can hope to avoid the Extension Problem.

In particular over the last 30 or so years, many problems from a variety of areas of mathematics have been translated into problems about finite groups, and then solved via this reduction process using the classification of the finite simple groups together with information about the simple groups.

Here is one example from my own work. Universal algebraists would like to describe finite lattices as lattices of congruences of finite algebras. An old result of Palfy and Pudlak [PP] says that each finite lattice is of this sort if and only if each finite lattice is an interval in the subgroup lattice of some finite group. The general consensus among simple group theorists is that the answer to this question is negative, and indeed John Sharesian has a conjecture in [S] of what counterexamples should look like, and has suggested a particular class of such lattices. In [A2], I've reduced the problem of showing that Sharasian's lattices are not intervals to two problems about the lattice of overgroups of subgroups of almost simple groups. (A finite group $G$ is almost simple if it has a unique minimal normal subgroup $L$, and $L$ is nonabelian simple.) Work is in progress to solve the two problems, using our knowledge of the subgroup structure of almost simple groups.

The two most important types of information about simple groups $G$ required to achieve and take advantage of general reductions of this kind are:

(I) the permutation representations of $G$ on sets, or equivalently the study of the subgroup structure of $G$, and

(II) the linear representations of $G$ on vector spaces; the case where the field of definition is finite is of particular importance.

Thus these days finite group theorists devote a lot of time and effort to the study of such representations of (nearly) simple groups.

\section{Classifying the Finite Simple Groups}

Next we consider the finite simple groups and their classification. For more details on this subject see [ALSS].
Classification Theorem. Each finite simple group is isomorphic to one of the following:

(1) A group of prime order.

(2) An alternating group.

(3) A group of Lie type.

(4) One of 26 sporadic simple groups.

The groups of prime order are the abelian simple groups.

The alternating group on a finite set $X$ is a normal subgroup of index 2 in the symmetric group on $X$. The symmetric group is the automorphism group of $X$ in the category of sets, so the alternating group is essentially the automorphism group of this very nice object.

The groups of Lie type are linear groups: groups of automorphisms of some family $X$ of polynomial functions on some finite dimensional vector space. A group of Lie type can be viewed as a form of a simple algebraic group.

The sporadic groups live in no known naturally defined infinite family of simple groups.

The theorem supplies a representation of each group in the first three cases as (essentially) the group of automorphisms of some nice object $X$, which can be used effectively to study the group. Some of the sporadics also have such representations, but others are only studied using local group theory and the classification itself.

So, how does one go about classifying simple groups? The primary tool is the local theory of finite groups. Let $G$ be a finite group and $p$ a prime. A $p$-local subgroup of $G$ is the normalizer of a nontrivial $p$-subgroup of $G$. In the classification, the focus is on the 2-locals. To oversimplify a bit, the simple groups are classified in terms of their 2-local structure.

The classification proceeds by induction on the group order. Let $\mathcal{K}$ be the list of simple groups appearing in the statement of the classification theorem. Define a finite group $G$ to be a $\mathcal{K}$-group if each simple section of $G$ is in $\mathcal{K}$. (A section of $G$ is a group of the form $A / B$ for some $B \unlhd A \leq G$.) In attempting to prove the theorem, we consider a simple group $G$ of minimal order subject to $G$ not in $\mathcal{K}$. Then each proper section of $G$ is a $\mathcal{K}$-group. This $\mathcal{K}$-group condition is used extensively in the proof of the theorem. For such appeals to be effective, we need many properties of the groups in $\mathcal{K}$.

Here are three questions to consider:

Question 1. What do local subgroups of groups in $\mathcal{K}$ look like? How do they differ from the locals in a random finite group?

Question 2. How do we force the local structure of our counterexample $G$ to resemble that of some $\bar{G}$ in $\mathcal{K}$ ? 
Question 3. If $G$ and $\bar{G}$ have similar local structure, how do we prove that $G \cong \bar{G}$ ?

I'll focus on Quesions 1 and 2.

At this point need to introduce a few concepts and a little technical notation and terminology. Let $L$ be a finite group. Write $O_{2}(L)$ for the largest normal 2-subgroup of $L$ and write $O(L)$ for the largest normal subgroup of $L$ of odd order. Call $O(L)$ the core of $L$. We will see that cores appear as obstructions in many parts of the proof of the classification.

A component of $L$ is a subnormal quasisimple subgroup of $L$. (A group $K$ is quasimple if $K=[K, K]$ is the commutator group of $K$ and $K / Z(K)$ is simple.) Think of the components of $L$ as normal nonabelian simple subgroups of $L$.

Define $L$ to be of characteristic 2-type if for each 2-local subgroup $H$ of $L$, we have $C_{H}\left(O_{2}(H)\right) \leq$ $\mathrm{O}_{2}(H)$. That is all "small" normal subgroups of $H$ are 2-groups and $\mathrm{O}_{2}(\mathrm{H})$ dominates the structure of $H$. Each group of Lie type over a field of characteristic 2 is of characteristic 2-type, so we should think of groups of characteristic 2-type as even groups.

Define $L$ to be of component type if for some involution $t$ in $L, C_{L}(t) / O\left(C_{L}(t)\right)$ has a component. Almost all groups of Lie type over fields of odd characteristic are of component type, so we should think of groups of component type as odd groups. Centralizers of involutions in alternating groups and sporadic groups have some involutions centralizers of one type and some of the other.

Gorenstein-Walter Dichotomy Theorem. If $L$ is simple and not "too small" then $L$ is either of characteristic 2-type or component type.

In this theorem $G$ is "small" if it is of 2-rank at most 2. After treating the "small" simple groups, the proof of the classification proceeds by considering each of the two cases in the Dichotomy Theorem (the even and odd groups), and treats them with different methods. In this article I'll concentrate on the groups of component type, since that is the portion of the proof the program I mentioned in the introduction seeks to simplify. So our focus will be on the odd groups.

Assume then that our minimal counter example $G$ is of component type. Recall this means that for some involution $t$ in $G, C_{G}(t) / O\left(C_{G}(t)\right)$ has a component. But if $\bar{G}$ in $\mathcal{K}$ is of component type then more is true: $C_{\bar{G}}(\bar{t})$ has a component. This suggests:

B-Conjecture. If $L$ is a finite group with $O(L)=1$ and $t$ is an involution in $L$, then each component of $C_{L}(t) / O\left(C_{L}(t)\right)$ is the image of a component of $C_{L}(t)$.

If $G$ satisfies the condition in the B-Conjecture then it is possible to play combinatorial games and show that if $t$ is chosen so that a component $K$ of $C_{G}(t)$ is "large" among all choices for $t$ and $K$, then $C_{G}(t)$ re- sembles the centralizer of some involution in some member of $\mathcal{K}$; indeed the structure of $C_{G}(t)$ is dominated by, and can be retrieved from, $K$. In this case we say $K$ is a standard subgroup of $G$. Then one must go on and solve the:

Standard Form Problem for K. Given a quasisimple group $K$ show that each simple group in which $K$ is standard is in $\mathcal{K}$.

To implement this approach, one must first verify the B-Conjecture. Unfortunately the proof of the $\mathrm{B}$-Conjecture is long and unpleasent. Note the obstruction posed by the B-Conjecture arises from the cores of 2-locals, or more generally the cores of finite groups. Perhaps the main reason to believe that the groups of component type are best treated from the point of view of 2-fusion systems is that 2-fusion systems have no cores.

We will return to groups of component type in a while, but first a few words about fusion systems.

\section{Fusion Systems}

The notion of a "fusion system" was introduced by Luis Puig (cf. [P]) who also proved many of the most basic theorems about fusion systems. However we will use different notation and terminology (introduced by topologists) than Puig. Our basic reference for fusion systems will be [AKO].

Let $p$ be a prime and $S$ a finite $p$-group. A fusion system on $S$ is a category $\mathcal{F}$ whose objects are the subgroups of $S$ and such that the set $\operatorname{hom}_{\mathcal{F}}(P, Q)$ of morphisms from a subgroup $P$ to a $\operatorname{subgroup} Q$ consists of injective group homomorphisms from $P$ into $Q$, and such that these morphism sets satisfy two weak axioms. In addition $\mathcal{F}$ is saturated if two more axioms are satisfied. See section I.2 in [AKO] for the precise definitions; rather than giving them here, it is perhaps more illuminating to consider the following example:

Standard Example. Let $G$ be a finite group and $S$ a Sylow $p$-subgroup of $G$. Write $\mathcal{F}_{S}(G)$ for the fusion system on $S$ such that hom $(P, Q)$ consists of the maps from $P$ into $Q$ induced by conjugation in $G$ : that is the maps $c_{g}: P \rightarrow Q$, where $c_{g}: x \mapsto g^{-1} x g$ for $g \in G$ with $g^{-1} P g \leq Q$ and $x \in P$.

Call $\mathcal{F}_{S}(G)$ the $p$-fusion system of $G$. Elements $x$ and $y$ in $S$ are said to be fused in $G$ if $x$ and $y$ are conjugate in $G$. The study of fusion in finite groups goes back at least to Burnside and played an important role in the original proof of the classification, although at that time the formal notion of a fusion system did not exist.

Using Sylow's Theorem, one can verify that the $p$-fusion system of a finite group is saturated. However there do exist saturated fusion systems that do not come from groups; such systems are said to be $e x$ - 
otic. For example there is one known infinite family of simple exotic 2-fusion systems: the Benson-Solomon systems.

It is possible to translate many (but not all) concepts from local finite group theory to analogous notions for fusion systems. Sometimes these notions behave better for fusion systems, and difficulties which arise for groups vanish for fusion systems. The obstruction corresponding to the B-Conjecture is an important example of this effect.

Fusion systems can be regarded as algebraic or topological objects. In particular algebraic topologists are interested in fusion systems for their own reasons. Another advantage to working with fusion systems is that arguments from algebraic topology become available, or at least are more intuitive for fusion systems than for groups.

Finally Puig's primary area of interest is the modular representation theory of finite groups. Puig created his theory as a tool in modular representation theory, where it has come to be important. Thus fusion systems are of interest in at least three mathematical specialties: modular representation theory, algebraic topology, and the local theory of finite groups.

We will need some basic notation, terminology, and concepts for fusion systems. Let $\mathcal{F}$ be a fusion system on a finite $p$-group $S$. For $P \leq S$, let $P^{\mathcal{F}}=\{P \phi$ : $\left.\phi \in \operatorname{hom}_{\mathcal{F}}(P, S)\right\}$ be the set of conjugates of $P$. We say that $P$ is fully normalized in $\mathcal{F}$ if $\left|N_{S}(P)\right| \geq\left|N_{S}(Q)\right|$ for each $Q \in P^{\mathcal{F}}$. In the Standard Example, $P$ is fully normalized if and only if $N_{S}(P) \in \operatorname{Syl}_{p}\left(N_{G}(P)\right)$. Write $\mathcal{F}^{f}$ for the set of fully normalized subgroups of $S$.

Define the normalizer in $\mathcal{F}$ of $P$ to be the subsystem $N_{\mathcal{F}}(P)$ of $\mathcal{F}$ on $N_{S}(P)$ such that for $Q, R \leq N_{S}(P)$ an $\mathcal{F}$-morphism $\phi: Q \rightarrow R$ is a $N_{\mathcal{F}}(P)$-morphism if $\phi$ extends to an $\mathcal{F}$-morphism $\hat{\phi}: P Q \rightarrow P R$ acting on $P$. Define $P$ to be normal in $\mathcal{F}$ and write $P \unlhd \mathcal{F}$ if $\mathcal{F}=N_{\mathcal{F}}(P)$.

One can define the centralizer $C_{\mathcal{F}}(P)$ of $P$ in $\mathcal{F}$ similarly. The following result of Puig appears as II.2.1 in [AKO]:

Theorem 3.1 (Puig). If $\mathcal{F}$ is saturated and $P \in \mathcal{F}^{f}$ then $N_{\mathcal{F}}(P)$ and $C_{\mathcal{F}}(P)$ are saturated.

There is a largest subgroup $O_{p}(\mathcal{F})$ normal in $\mathcal{F}$. We say that $\mathcal{F}$ is constrained if $C_{S}\left(O_{p}(\mathcal{F})\right) \leq O_{p}(\mathcal{F})$.

The local subsystems of $\mathcal{F}$ are the subsystems $N_{\mathcal{F}}(P)$ for $P \leq S$. These local subsystems are the analogues of the local subgroups in a finite group; they allow us to build a local theory of fusion systems. To take advantage of Puig's theorem, we need the following result, which is part of I.2.6 in [AKO].

Lemma 3.2. Let $\mathcal{F}$ be saturated and $P \leq S$. Define $\mathfrak{A}(P)=\left\{\phi \in \operatorname{hom}_{\mathcal{F}}\left(N_{S}(P), S\right): P \phi \in \mathcal{F}^{f}\right\}$. Then there exists $Q \in P^{\mathcal{F}} \cap \mathcal{F}^{f}$ and for each such $Q$ there exists $\alpha \in \mathfrak{A}(P)$ with $P \alpha=Q$.

\section{Groups of Component Type}

Assume $G$ is a finite group with $O(G)=1$ such that $G$ satisfies the B-Conjecture:

B-Conjecture. If $G$ is a finite group such that $O(G)=$ 1 then for each involution $t$ in $G$, each component of $C_{G}(t) / O\left(C_{G}(t)\right)$ is the image of a component of $C_{G}(t)$.

The B-Conjecture was proved by brute force. One major reason why one could hope that the theory of fusion systems might make possible a simplification of this part of the classification is that it should allow us to avoid having to prove the B-Conjecture.

Let $\mathfrak{C}$ be the set of components of centralizers of involutions of $G$.

Component Theorem. If $L$ is "maximal" in $\mathfrak{C}$ then (essentially) either

(1) $L$ is a component of $G$, or

(2) $L$ is standard in $G$.

Here $L$ is standard in $G$ if $K=C_{G}(L)$ is tightly embedded in $G, L \unlhd N_{G}(K)$, and $L$ commutes with none of its conjugates.

A subgroup $K$ of $G$ is tightly embedded in $G$ if $K$ is of even order but $K \cap K^{g}$ is of odd order for each conjugate $K^{g} \neq K$.

Theorems on tight embedding show that if $L$ is standard in $G$ then either a Sylow 2-subgroup $Q$ of $K$ contains a unique involution, or $Q$ is elementary abelian; in practice in the latter case $|Q| \leq 4$. At this point the centralizer in $G$ of an involution of $Q$ looks like the centralizer in some known simple group, and it remains to solve the:

Standard Form Problem for L. Determine those finite groups $G$ with $O(G)=1$ possessing a standard subgroup isomorphic to $L$.

Definition (Gorenstein). $L \in \mathfrak{C}$ is terminal in $G$ if for each involution $t \in C_{G}(L), L$ is a component of $C_{G}(t)$.

Example. If $L$ is a component of $G$ then $L$ is terminal in $G$.

Lemma 4.1. If $L$ is terminal and commutes with none of its conjugates then $L$ is standard in $G$.

Proof. Let $K=C_{G}(L)$. First, if $g \in G$ and $\left|K \cap K^{g}\right|$ is even then there is an involution $t \in K \cap K^{g}$. Then $L$ and $L^{g}$ are components of $C_{G}(t)$, so as $\left[L, L^{g}\right] \neq 1, L=L^{g}$ and hence $K=K^{g}$. Therefore $K$ is tightly embedded in $G$.

Second, if $g \in N_{G}(K)$ then we just saw that $L=L^{g}$, so indeed $L \unlhd N_{G}(K)$.

Thus to prove the Component Theorem it suffice to:

(a) prove $L$ is terminal, and then

(b) show if $\left[L, L^{g}\right]=1$ for some $g \in G$ then $L$ is a component of $G$. 
I close with two examples that we want to avoid, as the corresponding standard form problems can be difficult:

Example 4.2. Let $G=\left(L_{1} \times L_{2}\right)\langle t\rangle$ be a wreath product of a simple group $L_{1} \cong L_{2}$ by a group $\langle t\rangle$ of order 2 ; ie. $L_{1}^{t}=L_{2}$. Then $L=\left\{l l^{t}: l \in L_{1}\right\} \cong L_{1}$ is a standard subgroup of $G$.

Example 4.3. Let $G=X\langle t\rangle$ where $X=X\left(q^{2}\right)$ is of Lie type over $\mathbf{F}_{q^{2}}$ with $q$ even, and $t$ is an involution inducing a field automorphism on $X$. Then (essentially) $C_{G}(t)=\langle t\rangle \times L$ with $L \cong X(q)$ standard in $G$.

These examples are difficult because the 2-share $n_{t}$ of $\left|C_{G}(t)\right|$ is small relative to the 2-share $n_{G}$ of $|G|$; roughly $n_{G}=n_{t}^{2}$. This makes it hard to retrieve $G$ from $C_{G}(t)$.

As the groups in the two examples are of component type, they are "odd groups" from the point of view of the original proof of the classification. We wish to move the boundary between even and odd groups so that the groups in these examples need never be considered from the point of view of the centralizer of $t$.

\section{2-Fusion Systems of Component Type}

In this section let $\mathcal{F}$ be a saturated fusion system on a finite 2-group $S$.

A subgroup $P$ of $S$ is strongly closed in $S$ with respect to $\mathcal{F}$ if for each $Q \leq P$ and $\phi \in \operatorname{hom}_{\mathcal{F}}(Q, S)$, we have $Q \phi \leq P$.

From section I.6 in [AKO], we have the notion of a normal subsystem of $\mathcal{F}$, and from section II.5 in [AKO], for each $T \leq S$ strongly closed in $S$ with respect to $\mathcal{F}$, we have a factor system $\mathcal{F} / T$. If $\mathcal{E} \unlhd \mathcal{F}$ and $T$ is Sylow in $\mathcal{E}$, then $T$ is strongly closed, so we can define $\mathcal{F} / \mathcal{E}=$ $\mathcal{F} / T$.

Then we can define simple systems and subnormal subsystems of $\mathcal{F}$ in the obvious way by analogy with groups. There is also a smallest normal subsystem $O^{2}(\mathcal{F})$ such that $\mathcal{F} / O^{2}(\mathcal{F})$ is a 2-group.

Define $\mathcal{F}$ to be quasisimple if $\mathcal{F}=O^{2}(\mathcal{F})$ and $\mathcal{F} / Z(\mathcal{F})$ is simple. The components of $\mathcal{F}$ are its subnormal quasisimple subsystems. These components have properties similar to those of components of groups.

Define $\mathcal{F}$ to be of component type if for some fully centralized involution $t$ (ie. $\left.\langle t\rangle \in \mathcal{F}^{f}\right), C_{\mathcal{F}}(t)$ has a component. Define $\mathcal{F}$ to be of characteristic 2-type if for each $P \in \mathcal{F}^{f}, N_{\mathcal{F}}(P)$ is constrained (cf. section 3).

The following result is II.14.3 in [AKO]; it should be compared to the Dichotomy Theorem for groups in section 2, whose statement is less attractive and whose proof is much more difficult. Also the pres- ence of cores in the definition of "groups of component type" makes the Dichotomy Theorem for groups hard to apply, while cores are invisible in the 2-fusion systems of groups (cf. Remark 6.1) so such difficulties vanish when working with fusion systems.

Dichotomy Theorem for Fusion Systems. $\mathcal{F}$ is either of component type or characteristic 2-type.

Remark 5.1. We can only define the notion of a "component" in a saturated system, and (from Theorem 3.1), in the definition of systems of component type, to insure that $C_{\mathcal{F}}(t)$ is saturated, we need the condition $t \in \mathcal{F}^{f}$.

In a group $G$ we defined $\mathfrak{C}(G)$ to be the set of components of centralizers of involutions of $G$. What is the right definition of $\mathfrak{C}(\mathcal{F})$ ?

Recall from Lemma 3.2 that for $P \leq S, \mathfrak{A}(P)$ denotes the set of $\alpha \in \operatorname{hom}_{\mathcal{F}}\left(N_{S}(P), S\right)$ such that $P \alpha \in \mathcal{F}^{f}$, and that for each $Q \in P^{\mathcal{F}} \cap \mathcal{F}^{f}$ there is $\alpha \in \mathfrak{A}(P)$ with $P \alpha=Q$.

A subsystem $\mathcal{E}$ of $\mathcal{F}$ centralizes $P \leq S$ if $\mathcal{E} \leq C_{\mathcal{F}}(P)$. Given a quasisimple subsystem $\mathcal{C}$ of $\mathcal{F}$, define $\mathcal{I}(\mathcal{C})$ to consist of the involutions $t$ centralizing $\mathcal{C}$ such that for $\alpha \in \mathfrak{A}(t), \mathcal{C} \alpha$ is a component of $C_{\mathcal{F}}(t \alpha)$. Then define $\mathfrak{C}=\mathfrak{C}(\mathcal{F})$ to be the set of quasisimple subsystems $\mathcal{C}$ of $\mathcal{F}$ such that $\mathcal{I}(\mathcal{C}) \neq \varnothing$. We decree that $\mathfrak{C}$ is the set of components of involution centralizers in $\mathcal{F}$. In particular $\mathcal{F}$ is of component type iff $\mathfrak{C}(\mathcal{F}) \neq \varnothing$.

Remark 5.2. Given a general subsystem $\mathcal{E}$ of $\mathcal{F}$, the notions of the "normalizer" and "centralizer" in $\mathcal{F}$ of $\mathcal{E}$ are not defined (at least at present). But if $\mathcal{E}$ is a component of $\mathcal{F}$ then the two notions can be defined.

Let $\mathcal{C} \in \mathfrak{C}$ with $T$ Sylow in $\mathcal{C}$ and $t \in \mathcal{I}(\mathcal{C})$. Define $Q_{t}=\left(C_{S}(t) \alpha \cap C_{C_{S}(t \alpha)}(\mathcal{C} \alpha)\right) \alpha^{-1}$ for $\alpha \in \mathfrak{A}(t)$.

Two subsystems $\mathcal{E}_{i}$ of $\mathcal{F}$ with Sylow groups $T_{i}$, for $i=1,2$, are said to centralize each other if $\mathcal{F}$ contains a central product $\mathcal{E}_{1} * \mathcal{E}_{2}$ of the systems; equivalently $T_{i}$ centralizes $\mathcal{E}_{3-i}$ for $i=1,2$.

Define $\Delta(\mathcal{C})$ to consist of those conjugates $\mathcal{C}_{1}$ of $\mathcal{C}$ such that for $T_{1}$ Sylow in $\mathcal{C}_{1}, \mathcal{C}$ centralizes $\mathcal{C}_{1}$, each involution in $T_{1}$ is in $\mathcal{I}(\mathcal{C})$, and each involution in $T$ is in $\mathcal{I}\left(\mathcal{C}_{1}\right)$. Set $\mathcal{C}^{\perp}=\Delta(\mathcal{C}) \cup\{\mathcal{C}\}$.

The following result appears in the preprint [A3]:

Theorem 5.3. If $\mathcal{C}$ is "maximal" in $\mathfrak{C}, T \in \mathcal{F}^{f}$, and $\mathcal{C}^{\perp} \neq$ $\{\mathcal{C}\}$ then either

(1) $\mathcal{C}$ is a component of $\mathcal{F}$, or

(2) $T$ has a unique involution, $\mathcal{C}^{\perp}=\left\{\mathcal{C}, \mathcal{C}_{1}\right\}$ is of order 2 , and $Z(\mathcal{C})=Z\left(\mathcal{C}_{1}\right)$.

Define $\mathcal{C}$ to be terminal in $\mathfrak{C}$ if $\mathcal{C}=\mathcal{C}^{\perp}$ and for each $t \in \mathcal{I}(\mathcal{C})$ and each involution $i \in Q_{t}$, we have $i \in \mathcal{I}(\mathcal{C})$. Notice that this definition is analogous to the Gorenstein definition for groups in section 4, except that we are adding the condition that $\mathcal{C}$ "commutes with none of its conjugates". 
Theorem 5.4. If $\mathcal{C}$ is "maximal" in $\mathfrak{C}$ and $T \in \mathcal{F}^{f}$ then (essentially) either $\mathcal{C}$ is a component of $\mathcal{F}$ or $\mathcal{C}$ is terminal in $\mathfrak{C}$.

Proof. This is also a result in the preprint [A3]. Some other cases also arise, but this simplified statement captures the spirit of the result.

Theorem 5.4 is analogous to the Component Theorem for groups, except it is weaker: we've only shown that $\mathcal{C}$ is terminal, not that $\mathcal{C}$ is standard. On the other hand in the case of groups, if $L$ were terminal and commuted with no conjugate then from Lemma 4.1, $L$ would be standard.

But what does it mean for $\mathcal{C}$ to be "standard"?

Recall that a quasisimple subgroup $L$ of a group $G$ is standard in $G$ if $K=C_{G}(L)$ is tightly embedded in $G, L \unlhd N_{G}(K)$, and $L$ commutes with none of its conjugates.

It is possible to define the notion of a tightly embedded subsystem of $\mathcal{F}$, and to prove theorems about tightly embedded subsystems analogous to the corresponding theorem about tightly embedded subgroups. But recall we don't have a notion of the "centralizer" in $\mathcal{F}$ of $\mathcal{C}$, nor a notion of the "normalizer" in $\mathcal{F}$ of a centralizer.

Nevertheless I've advanced a definition of a "standard subsystem" of $\mathcal{F}$ that involves four conditions on $\mathcal{C}$. I'll only mention the first condition, Condition (S1). Write $\tilde{\mathcal{X}}(\mathcal{C})$ for the set of 2-subgroups $P$ of $\mathcal{F}$ centralizing $\mathcal{C}$ such that each involution in $P$ is in $\mathcal{I}(\mathcal{C})$. For example if $\mathcal{C}$ is terminal and $t \in \mathcal{I}(\mathcal{C})$, then $Q_{t} \in \tilde{\mathcal{X}}(\mathcal{C})$. Condition $(\mathrm{S} 1)$ says that $\tilde{\mathcal{X}}(\mathcal{C})$ contains a unique maximal member $Q$.

It can be shown that, if $\mathcal{C}$ is standard in $\mathcal{F}$, then $Q$ is Sylow in a saturated subsystem $\mathcal{Q}$ of $\mathcal{F}$ centralizing $\mathcal{C}$, such that $\mathcal{Q}$ is tightly embedded in $\mathcal{F}$ and $\mathcal{C} \unlhd N_{\mathcal{F}}(Q)$. We decree that $\mathcal{Q}$ is the centralizer in $\mathcal{F}$ of $\mathcal{C}$.

Given a standard subsystem $\mathcal{C}$ in $\mathcal{F}$, one can then (just as in the case of groups) use the theorems on tightly embedded subsystems to show that either $Q$ is elementary abelian or $Q$ has a unique involution. Then the centralizer of an involution in $Q$ resembles the centralizer in some known simple system, and it remains to solve the corresponding standard form problem for $\mathcal{C}$.

There is an exception to this approach. If $\mathcal{C}$ is the 2 -fusion system of a group of Lie type in odd characteristic other than $L_{2}(q)$, one instead seeks to prove a version for 2-fusion systems of the so-called Classical Involution Theorem for groups in [A1], and extend that result to a fusion theoretic version of a theorem of Walter in [W]. This would allow us to avoid treating the standard form problems for most of the 2-fusion systems of groups of Lie type in odd characteristic other than $L_{2}(q)$.

Caution. If $\mathcal{C}$ is terminal then, in general, $\mathcal{C}$ need not be standard. For if $x \in Q$ and $s \in C_{S}(T)$ then $x^{s} \in Q$ by the uniqueness of $Q$ in (S1), so $z=x x^{s} \in Q$. Then if $1 \neq$ $z \in T$, we have $z \in Z(\mathcal{C})$, so in particular $Z(\mathcal{C}) \neq 1$. But in Examples 4.2 and 4.3, taking $\mathcal{F}=\mathcal{F}_{S}(G)$ and $\mathcal{C}=\mathcal{F}_{T}(L)$, we have $\mathcal{C}$ terminal with $Z(\mathcal{C})=1$, whereas $C_{S}(T) \not \leq C_{S}(t)$.

On the other hand if $\mathcal{C}$ is terminal and $Z(\mathcal{C}) \neq 1$ then the first three condition of the definition of "standard subsystem" are satisfied, and, in the examples I've looked at, the fourth condition can be established via ad hoc means.

Thus we seek to avoid the "bad" cases in Examples 4.2 and 4.3, just as we wished to avoid them for groups. Note that these cases do not arise when $Z(\mathcal{C}) \neq 1$. We will see one way to avoid these cases in a moment; but first we need to take a closer look at the "known" simple 2-fusion systems.

\section{Simple 2-Fusion Systems}

Write $\mathcal{K}$ for the class of "known" simple 2-fusion systems.

The only exotic members of $\mathcal{K}$ are the BensonSolomon systems, which are of component type. Indeed if $\mathcal{F}$ is Benson-Solomon then $\mathcal{F}$ has one class of involutions $t^{\mathcal{F}}$, and $C_{\mathcal{F}}(t)$ is the 2-fusion system of $\operatorname{Spin}_{7}(q)$ for some odd prime power $q$.

Thus all other members $\mathcal{F}$ of $\mathcal{K}$ are of the form $\mathcal{F}_{S}(G)$ for some finite group $G$ and $S \in \mathrm{Syl}_{2}(G)$. Assume $\mathcal{F}$ is not the system of the group of order 2 .

Remark 6.1. Let $G^{*}=G / O(G)$. Then the projection $*: S \rightarrow S^{*}$ is an isomorphism of $\mathcal{F}_{S}(G)$ with $\mathcal{F}_{S^{*}}\left(G^{*}\right)$. That is $O(G)$ does not effect 2-fusion in $G$.

Remark 6.2. If $H \unlhd G$ then $\mathcal{F}_{S \cap H}(H) \unlhd \mathcal{F}$.

Remark 6.3. $O_{2}(G) \leq O_{2}(\mathcal{F})$.

We wish to show that $\mathcal{F}=\mathcal{F}_{S}(G)$ is the 2-fusion system of some simple group $G$. By Remark 6.1, replacing $G$ by $G / O(G)$, we may assume $O(G)=1$. As $\mathcal{F}$ is simple and not of order 2, we conclude from Remark 6.3 that $O_{2}(G)=1$. Let $L$ be a minimal normal subgroup of $G$. By Remark 6.2 and the simplicity of $\mathcal{F}, S \leq L$ and $\mathcal{F}=\mathcal{F}_{S}(L)$, so we may take $G=L$. As $O_{2}(G)=O(G)=1, L$ is the direct product of isomorphic nonabelian simple groups. Then by Remark 6.2, $L$ is simple. Thus we've shown that each member of $\mathcal{K}$ that is not Benson-Solomon is of the form $\mathcal{F}_{S}(G)$ for some nonabelian simple group $G$.

What about the converse?

A Goldschmidt group is a nonabelian simple group $G$ such that $G$ has a nontrivial abelian 2-subgroup strongly closed in a Sylow 2-subgroup $S$ of $G$ with respect to $G$. By a theorem of Goldschmidt [Go], $G$ is Goldschmidt iff $G$ is of Lie type in characteristic 2 of Lie rank 1, or $S$ is abelian.

Remark 6.4. If $G$ is Goldschmidt then $S \unlhd G$. In particular the 2-fusion system of a Goldschmidt group is not simple. 
Remark 6.5. It turns out that if $G$ is a known simple group but not Goldschmidt then $\mathcal{F}$ is simple. Thus the members of $\mathcal{K}$ are the Benson-Solomon systems together with the 2-fusion systems of the known simple groups that are not Goldschmidt.

A member $\mathcal{C}$ of $\mathcal{K}$ is said to be tamely realized by some simple group $G=K(\mathcal{C})$ if (essentially) $\mathcal{C}=\mathcal{F}_{T}(G)$ and all automorphisms of $\mathcal{C}$ are induced by automorphisms of $G$. In particular, in as yet unpublished work, Broto, Moller, and Oliver show that most members of $\mathcal{K}$ that are not Benson-Solomon are tamely realized by some $K(\mathcal{C})$. This fact is crucial in analyzing systems of component type in an inductive setting, as it allows us to describe suitable extensions of $\mathcal{C} \in \mathfrak{C}$ induced in $\mathcal{F}$.

Define $\mathcal{F}$ to be a $\mathcal{K}$-system if each simple section of $\mathcal{F}$ is in $\mathcal{K}$. (A section of $\mathcal{F}$ is a factor system of a subsystem of $\mathcal{F}$.) If we attempt to classify all simple 2-fusion systems via an inductive approach, then a minimal counter example $\mathcal{F}$ to such a classification has the property that all proper sections of $\mathcal{F}$ are $\mathcal{K}$-systems.

In attempting to classify simple systems of component type (or some subclass of such systems) we may want to assume a weaker condition; for example for each $\mathcal{C} \in \mathfrak{C}, \mathcal{C} / Z(\mathcal{C}) \in \mathcal{K}$. Indeed we are led to impose further constraints on the members of $\mathfrak{C}$.

Let $\mathcal{C}$ be quasisimple on $T$. Define $\mathcal{C}$ to be intrinsic if $Z(\mathcal{C}) \neq 1$. The intrinsic $\mathcal{K}$-systems consist of the 2 -fusion systems of coverings of groups of Lie type and odd characteristic, alternating groups, and a finite number of groups of Lie type and characteristic 2 and sporadic groups.

If $\mathcal{C} \in \mathfrak{C}$, define $\mathcal{C}$ to be intrinsic in $\mathfrak{C}$ if $\mathcal{I}(\mathcal{C}) \cap$ $Z(\mathcal{C}) \neq \varnothing$.

Define $\mathcal{C}$ to be subintrinsic if there exists a member of $\mathfrak{C}(\mathcal{C})$ intrinsic in $\mathfrak{C}(\mathcal{C})$. The subinstrinsic members of $\mathcal{K}$ consist of the Benson-Solomon systems, most groups of Lie type in odd characteristic, and some sporadic groups.

If $\mathcal{C} \in \mathfrak{C}$, define $\mathcal{C}$ to be subinstrinsic in $\mathfrak{C}$ if there exists $\mathcal{D} \in \mathfrak{C}(\mathcal{C})$ such that $\mathcal{D}$ is intrinsic in $\mathfrak{C}(\mathcal{F})$.

Problem A. Assume $\mathcal{F}$ is simple and for each $\mathcal{C} \in \mathfrak{C}$, $\mathcal{C} / Z(\mathcal{C}) \in \mathcal{K}$. Assume some member of $\mathfrak{C}$ is subintrinsic. Prove $\mathcal{F} \in \mathcal{K}$.

We have identified one subclass of the class of fusion systems of component type which it seems likely we can classify: the systems arising in Problem A where $\mathfrak{C}$ contains a subintrinsic member. We next consider a second class: the systems of J-component type.

Recall the 2-rank $m_{2}(G)$ of a finite group $G$ is the largest integer $m$ such that $G$ contains a direct product of $m$ copies of the group of order 2 .

Define $\mathcal{J}=\mathcal{J}(\mathcal{F})$ to be the set of involutions $t \in S$ such that $m_{2}\left(C_{S}(t)\right)=m_{2}(S)$. For $\mathcal{C} \in \mathfrak{C}$, set $\mathcal{J}(\mathcal{C})=\mathcal{J} \cap$
$\mathcal{I}(\mathcal{C})$. Define $\mathfrak{C}_{J}$ to consist of those $\mathcal{C} \in \mathfrak{C}$ such that $\mathcal{J}(\mathcal{C}) \neq \varnothing$.

Define $\mathcal{F}$ to be of J-component type if

(a) for each $\mathcal{C} \in \mathfrak{C}, \mathcal{C} / Z(\mathcal{C}) \in \mathcal{K}$, and

(b) no member of $\mathfrak{C}$ is subintrinsic, and

(c) $\mathfrak{C}_{J} \neq \varnothing$.

Define $\mathcal{C} \in \mathfrak{C}_{J}$ to be $J$-terminal if $\mathcal{C}^{\perp}=\mathcal{C}$ and for each $t \in \mathcal{J}(\mathcal{C})$, each involution in $Q_{t}$ is in $\mathcal{I}(\mathcal{C})$. The following result appears in the preprint [A3]:

Theorem 6.6. Assume $\mathcal{F}$ is of J-component type, $\mathcal{C} \in \mathfrak{C}_{J}$ is "maximal" in $\mathfrak{C}_{J}$, and $T \in \mathcal{F}^{f}$. Then one of the following holds:

(1) $K(\mathcal{C})$ is $A_{n}$ for some $n \geq 8$.

(2) $K(\mathcal{C})$ is $L_{2}(q)$ for some odd $q$, and many conditions hold.

(3) $K(\mathcal{C})$ is $L_{3}(4), G_{2}(4), L_{3}(3)$, or $U_{3}(3)$.

(4) $\mathcal{C}$ is a component of $\mathcal{F}$.

(5) $\mathcal{C}$ is J-terminal.

In the case where $\mathcal{C}$ is J-terminal, one would attempt to show that $\mathcal{C}$ is actually standard, and then go on to either obtain a contradiction or show $\mathcal{F} \in \mathcal{K}$. In the exceptional cases (1)-(3), one could attempt to do the same; for example I have notes treating case (1).

In any event we are led to the following problems:

Problem B. Assume $\mathcal{F}$ is simple of J-component type. Prove $\mathcal{F} \in \mathcal{K}$.

Define a simple system $\mathcal{F}$ to be odd if either

(A) for each $\mathcal{C} \in \mathfrak{C}, \mathcal{C} / Z(\mathcal{C}) \in \mathcal{K}$ and some member of $\mathfrak{C}$ is subintrinsic, or

(B) $\mathcal{F}$ is of J-component type.

Define $\mathcal{F}$ to be even if all proper sections of $\mathcal{F}$ are $\mathcal{K}$-systems and $\mathcal{F}$ is not odd.

Problem C. Prove all odd systems are in $\mathcal{K}$.

Problem D. Prove all even systems are in $\mathcal{K}$.

\section{From Fusion Systems to Groups}

We wish to use theorems about 2-fusion systems to prove theorems about groups. In particular we want to study groups $G$ in which every proper section of $G$ is a $\mathcal{K}$-group, as a minimal counter example to the theorem classifying the finite simple groups has this property. As a first step we have:

Theorem 7.1. Let $G$ be a finite group such that each proper section of $G$ is a $\mathcal{K}$-group. Let $\mathcal{F}$ be the 2-fusion system of $G$. Then either

(1) $G$ is a $\mathcal{K}$-group, or

(2) $G$ is simple, $\mathcal{F}=O^{2}(\mathcal{F})$, and $\mathcal{F}$ is almost simple.

Here $\mathcal{F}$ is almost simple if $\mathcal{F}$ has a unique minimal normal subsystem, and that subsystem is simple and not of order 2. 
The theorem shows that the 2-fusion system of our minimal counter example is essentially simple. Thus results about simple 2-fusion systems can indeed be useful in studying simple groups.

Let $G \in \mathcal{K}$ be a known simple group, or assume $G \notin \mathcal{K}$ but each proper section of $G$ is a $\mathcal{K}$-group. Let $\mathcal{F}$ be the 2-fusion system of $G$. Define $G$ to be odd if $\mathcal{F}$ is odd and $G$ to be even if $\mathcal{F}$ is even. (Actually when $G \notin \mathcal{K}$ we want the simple minimal normal subsystem of $\mathcal{F}$ to be odd or even.) What can we say about $G$ ? In the odd case, we want to use a solution to Problem C to show that $G \in \mathcal{K}$. I won't say anything more about that case, so instead assume $G$ is even.

Let $\mathcal{J}$ be the set of involutions $j \in G$ such that $m_{2}\left(C_{G}(j)\right)=m_{2}(G)$. Take $j \in \mathcal{J}$; conjugating in $G$, we may assume $j \in \mathcal{F}^{f}$. Then as $G$ is even, $C_{\mathcal{F}}(j)$ has no components, so the components of $C_{G}(j)^{*}=$ $C_{G}(j) / O\left(C_{G}(j)\right)$ are coverings of Goldschmidt groups. If $L^{*}$ is such a component and not simple then $L^{*} \cong$ $S L_{2}(q)$ for some odd $q$, or $L^{*}$ is a proper covering of $S z(8)$. The former can presumably be handled using the so-called Classical Involution Theorem [A1], and the latter using ad hoc methods. Thus we can presumably assume $G$ is $J$-locally Goldschmidt: for each $j \in \mathcal{J}(G)$, the components of $C_{G}(j) / O\left(C_{G}(j)\right)$ are Goldschmidt groups.

\section{Theorem 7.2. Assume G is J-locally Goldschmidt. Then}

(1) $O\left(C_{G}(t)\right)=1$ for each involution $t$ in $G$.

(2) If for some $j \in \mathcal{J}(G), C_{G}(j)$ has a component isomorphic to $L_{2}(q), q$ odd, $U_{3}(4),{ }^{2} G_{2}(q)$, or $J_{1}$, then $G \in \mathcal{K}$.

This leaves only the case where for $j \in \mathcal{J}(G)$, all components of $C_{G}(j)$ are isomorphic to $L_{2}\left(2^{n}\right), U_{3}\left(2^{n}\right)$, or $S z\left(2^{n}\right)$ with $n \geq 3$. Presumably in the case where such a component exists, it is not too difficult to obtain a contradiction. If so, we will have shown that for each $j \in \mathcal{J}(G), C_{G}\left(O_{2}\left(C_{G}(j)\right)\right) \leq O_{2}\left(C_{G}(j)\right)$.

We also known that if $t$ is an involution in $G$ and $L$ is a component of $C_{G}(t)$ with $T \in \operatorname{Syl}_{2}(G)$, then $\mathcal{F}_{T}(L)$ is not subintrinsic, so $L$ is of Lie type of even characteristic, an alternating group, or one of a small number of sporadic groups or groups of Lie type in odd characteristic of small Lie rank whose 2-fusion system is that of a group over $\mathbf{F}_{3}$.

Thus we see (in general terms) what a minimal counter example to the classification of finite simple groups that is even looks like, at least 2-locally. One could hope to handle this case using the GLS approach, or the approach of MSS. Alternatively, one could solve Problem D, and then use the fact that $\mathcal{F}$ is a known simple fusion system to show $G \in \mathcal{K}$. Perhaps the MSS methods could be used to solve Problem D.

\section{References}

[A1] M. Aschbacher, A characterization of Chevalley groups over fields of odd order, Annals Math. 106 (1977), 353-468.

[A2] M. Aschbacher, Signalizer lattices in finite groups, Michigan Math. J. 58 (2009), 79-103.

[A3] M. Aschbacher, Fusion systems of component type, preprint.

[AKO] M. Aschbacher, R. Kessar, and B. Oliver, Fusion Systems in Algebra and Topology, Cambridge University Press, 2011.

[ALSS] M. Aschbacher, R. Lyons, S. Smith, and R. Solomon, The Classification of the Finite Simple Groups; Groups of Characteristic 2 Type, Mathematical Surveys and Monographs, vol. 172, AMS, 2011.

[GLS1] D. Gorenstein, R. Lyons, and R. Solomon, The Classification of the Finite Simple Groups, Number 1, Mathematical Surveys and Monographs, vol. 40, AMS, 1994.

[Go] D. Goldschmidt, 2-fusion in finite groups, Ann. Math. 99 (1974), 70-117.

[P] L. Puig, Frobenius Categories Versus Brauer Blocks, Birkhauser, 2009.

[PP] P. Palfy and P. Pudlak, Congruence lattices of finite algebras and intervals in subgroup lattices of finite groups, Alg. Universalis 11 (1980), 22-27.

[S] J. Shareshian, Topology of order complexes of intervals in subgroup lattices, J. Alg. 268 (2003), 677-685.

[W] J. Walter, The B-Conjecture; Characterization of Chevalley groups, Memoirs AMS 61 (1986), 1-196. 